DOI

ARTICULO ORIGINAL

\title{
Tabaquismo y niveles de colesterol HDL en pacientes que asisten a una unidad de prevención secundaria cardiovascular
}

Darwin O. Cordovilla ORCID: 0000-0002-3572-7328

Postgrado de Reumatología. Facultad de Medicina. UdelaR.

Laura Llambí ORCID: 0000-0001-6636-792X Internista. Profesora Agregada. Clínica Médica A. Facultad de Medicina. UdelaR.

Selva Romero ORCID: 0000-0002-0989-726X Internista. Asistente Clínica Médica A. Facultad de Medicina. UdelaR.

\section{Smoking and HDL colesterol levels in patiens assisting a cardiovascular secundary prevention unit}

Tabagismo e níveis de colesterol HDL em pacientes atendidos em unidade de prevenção secundária cardiovascular

Resumen: Introducción: El tabaquismo es un importante factor de riesgo de enfermedad cardiovascular a nivel mundial y local. Los fumadores presentan niveles de colesterol HDL bajos. La cesación del tabaquismo eleva los niveles de HDL y reduce el riesgo cardiovascular. Objetivo: determinar la relación de los niveles de colesterol HDL en función del status fumador. Métodos: Estudio observacional, longitudinal, analítico, retrospectivo. La información fue tomada de historias clínicas de pacientes con cardiopatía isquémica revascularizados, con status de fumador y niveles de colesterol HDL asistidos en una Unidad de Prevención Secundaria. Los resultados se analizaron en frecuencias y medias. Se realizó chi-cuadrado para comparación de frecuencias y test de t de student para la comparación medias. Resultados: Se estudiaron 97 pacientes. El $48.5 \%$ eran fumadores, el $30 \%$ ex fumadores y el $22 \%$ no fumadores. El $58 \%$ de los fumadores activos, $57 \%$ de los fumadores en cesación y el $55 \%$ de los ex fumadores tenían valores de colesterol HDL bajo. A los seis meses: el $60 \%$ de los fumadores activos, el $40 \%$ de los fumadores en cesación y el $41 \%$ de los ex fumadores tenían valores de HDL bajo. Al comparar las frecuencias se obtuvo un valor de p 0,732 al inicio y un valor de p 0,311 a los 6 meses, no estadísticamente significativos. La media total de los valores de HDL al inicio fue de 40,72 mg/ $\mathrm{dl}$ y a los 6 meses de 43,42 mg/dl con un valor de p 0,003, estadísticamente significativo. Se comparó las medias de HDL inicial de los pacientes en cesación versus las medias de HDL de los ex fumadores a los 6 meses con un valor de p 0,054, que roza la significancia estadística. Conclusiones: Los niveles de colesterol HDL fueron bajos en los fumadores. Observamos que la cesación del tabaquismo provocó una tendencia al incremento delos niveles de colesterol HDL.

Palabras clave: tabaquismo, cese del tabaquismo, colesterol HDL

Abstract: Introduction: Smoking is an important risk factor for cardiovascular disease worldwide and locally. Smokers present low levels of HDL cholesterol. Cessation of smoking raises HDL levels and reduces cardiovascular risk. Objective: Determine the relationship of HDL cholesterol levels according to smoking status. Methods: An Observational, longitudinal, analytical, retrospective study. The information was taken from medical records of patients with revascularized ischemic heart disease, with smoker status and HDL cholesterol levels assisted at the Secondary Prevention Unit. The results were analyzed in frequencies and means. Chi-square was performed for comparison of frequencies and student's test for the means comparison. Results: 97 patients were studied, $48.5 \%$ were smokers, $30 \%$ were ex-smokers and $22 \%$ were non-smokers. The $58 \%$ of active smokers, $57 \%$ of smokers in cessation and $55 \%$ of ex-smokershad low values of HDL cholesterol. Six months later: $60 \%$ of active smokers, $40 \%$ of smokers in cessation and $41 \%$ of ex-smokers had low HDL values. When comparing frequencies, a value of $p 0.732$ was obtained at the beginning and a value of $p 0.3116$ months later, not statistically significant. The total mean HDL values at baseline were $40.72 \mathrm{mg} / \mathrm{dl}$ and at 6 months, $43.42 \mathrm{mg} / \mathrm{dl}$, with a p-value of 0.003 , statistically significant. The initial HDL mean of smokers in cessation was 
compared to the HDL mean of the ex-smokers at 6 months with a p-value 0.054 that borders on statistical significance. Conclusions: HDL levels were low in smokers. We observed that the cessation of smoking caused a tendency to increase HDL cholesterol levels.

Keywords: smoking, cessation of smoking, HDL cholesterol

Resumo: Introdução: O tabagismo é um importante fator de risco para doenças cardiovasculares em todo o mundo e localmente. Fumantes têm baixos níveis de colesterol HDL. A cessação do tabagismo aumenta os níveis de HDL e reduz o risco cardiovascular. Objetivo: determinar a relação dos níveis de colesterol HDL de acordo com o status de tabagismo. Métodos: Estudo observacional, longitudinal, analítico, retrospectivo. As informações foram retiradas de prontuários de pacientes com doença cardíaca isquêmica revascularizada, com status de fumante e níveis de colesterol HDL atendidos em uma Unidade de Prevenção Secundária. Os resultados foram analisados em frequências e médias. O qui-quadrado foi realizado para comparação de frequências e teste t de Student para comparação. Resultados: 97 pacientes foram estudados. $48,5 \%$ eram fumantes, $30 \%$ ex-fumantes e $22 \%$ não fumantes. $58 \%$ dos fumantes ativos, $57 \%$ dos fumantes em cessação e $55 \%$ dos ex-fumantes tinham baixos valores de colesterol HDL. Aos seis meses: $60 \%$ dos fumantes ativos, $40 \%$ dos fumantes em cessação e $41 \%$ dos ex-fumantes tinham baixos valores de HDL. Ao comparar frequências, um valor de $p$ 0,732 foi obtido no início e um valor de p 0,311 aos 6 meses, não estatisticamente significante. Os valores médios totais de HDL no início do estudo foram de 40,72 mg / dl e aos 6 meses, $43,42 \mathrm{mg} / \mathrm{dl}$ com um valor $\mathrm{p}$ de 0,003, estatisticamente significativo. Comparamos as médias iniciais do HDL dos pacientes que deixaram de fumar versus as médias do HDL dos ex-fumantes aos 6 meses, com um valor p de 0,054, o que limita a significância estatística. Conclusões: Os níveis de colesterol HDL foram baixos em fumantes. Observamos que a cessação do tabagismo causou uma tendência a aumentar os níveis de colesterol HDL.

Palavras-chave: tabagismo, cessação do tabagismo, colesterol HDL

Recibido: 22/07/2018 - Aceptado: 28/01/2019

Unidad de Tabaquismo y Unidad de Prevención Secundaria de la Clínica Médica "Ä" Hospital de Clínicas "Dr. Manuel Quintela”, primer piso, ala oeste. Facultad de Medicina. Universidad de la República (UdelaR). Montevideo, Uruguay. Correspondencia: E-mail: darwin_cordovilla@ hotmail.com / darwinoctavio.doc@gmail.com 


\section{Introducción}

Las Enfermedades Cardiovasculares (ECV) representan el 33\% a nivel mundial. En Uruguay, las ECV representan el $27 \%$ y son la primera causa de mortalidad con una tendencia descendente en los últimos años ${ }^{(1-3)}$.

El tabaquismo es responsable del $40 \%$ de las muertes por ECV a nivel mundial, mientras que en Uruguay representa el $14,7 \%$ del total de muertes anuales ${ }^{(4,5)}$. La Encuesta Nacional de Hogares Ampliada (ENHA) realizada en 2014, reporta que el $22,2 \%$ de la población uruguaya se declara fumadora. El $27 \%$ de los fumadores son hombres y el $17,9 \%$ son mujeres. El $45,8 \%$ de los fumadores se concentran en el grupo de edad comprendido entre 25 a 44 años, seguido por el grupo de 45 a 64 años con el 32\% (6). Uruguay en 2008 creó la Ley $N^{\circ} 18.256{ }^{(7)}$ para el control del consumo de tabaquismo que incluye políticas de publicidad, patrocinio, comercio y prohibición de fumar en lugares públicos con lo que se evidenció una tendencia descendente de fumadores, de $24.8 \%$ en 2006 a $22.2 \%$ en 2014. Esta encuesta además reporta que el $19,5 \%$ de los consultados dejó de fumar ${ }^{(6)}$. Esta ley se ve reflejada en la reducción del 17\% de ingresos hospitalarios por infarto de miocardio a los centros hospitalarios de Uruguay en los cuatro años posteriores de la ley ${ }^{(5)}$. Vásquez y cols evidenciaron que el $91,7 \%$ de los pacientes hospitalizados por cardiopatía isquémica fue derivado a una policlínica de cesación de tabaquismo de los cuales solo el $45,5 \%$ concurrió, y de estos el $68 \%$ estaba en cesación a los 12 meses de seguimiento ${ }^{(8)}$.

El tabaquismo se asocia con las ECV a través de múltiples mecanismos entre los que se incluyen: trastornos vasomotores, neurohumorales, disfunción hematológica, inflamación y aumento del estrés oxidativo. Fisiopatológicamente, el tabaquismo altera la liberación de catecolaminas, provocando una alteración de la peroxidación lipídica y liberación de ácidos grasos en forma de colesterol VLDL (very low density lipoproteins) y colesterol LDL (low density lipoproteins); esto provoca una disminución del colesterol HDL (high density lipoproteins) lo que favorece la aterogénesis vascular ${ }^{(9-17)}$. Las concentraciones de HDL se correlacionan negativamente con las enfermedades cardiovasculares, que es decir, cuanto menor es el HDL, mayor es el grado de la aterogénesis ${ }^{(15)}$.

La cesación del tabaquismo produce aumento de los niveles HDL, mientras que los niveles de triglicéridos (TG), LDL, colesterol total (CT) disminuyen o se mantienen sin cambios. El tiempo para la normalización de HDL se puede ver en poco tiempo, tres a cinco semanas y continuará aumentando siempre que se mantenga la cesación ${ }^{(9,15,18-20)}$.

En Uruguay desde el 2004 se desarrolla a través del Fondo Nacional de Recursos el Programa de Prevención Secundaria Cardiovascular, con el objetivo de optimizar el tratamiento de los pacientes con enfermedad coronaria, promover estilos de vida saludables, controlar los factores de riesgo cardiovascular y facilitar el acceso a la medicación cardioprotectora. El programa es aplicado en varios centros a través de las Unidades de Prevención Secundaria (UPS) conformadas por equipos multidisciplinarios quienes realizan el seguimiento y control de estos pacientes ${ }^{(21)}$.

Se ha determinado que el tabaquismo es uno de los principales factores de riesgo cardiovascular, debido a varios mecanismos, entre el que se destaca la disminución del colesterol HDL; y también se ha demostrado que la cesación del tabaquismo aumenta los valores de colesterol HDL. Sin embargo aún existe controversia y escasos datos nacionales de la relación entre el tabaquismo y niveles de HDL en pacientes con cardiopatía isquémica tratados con revascularización miocárdica. Se realizó el presente estudio con el objetivo de determinar la frecuencia de fumadores, así como los niveles medios de HDL según el status fumador. Pudiéndose observar el comportamiento de los valores del HDL en este grupo de pacientes.

\section{Metodología}

Se realizó un estudio observacional, longitudinal, descriptivo, retrospectivo de las historias clínicas electrónicas de los pacientes asistidos en una UPS, en el periodo comprendido entre 01/01/2011 a 31/12/2015, que cumplieron con los criterios de inclusión y exclusión. Los valores de lípidos plasmáticos se obtuvieron de los registros de paraclínica solicitados en los diferentes controles y que fueron volcados a dicho registro electrónico.

El estudio incluyó a pacientes mayores de 18 años y menores de 70 años, portadores de cardiopatía isquémica tratados con revascularización miocárdica por Angioplastia, Cirugía Cardíaca o fibrinolíticos y que contaban con registros séricos de HDL al ingreso a la UPS o 
al primer control coordinado. Se excluyó a pacientes sin registros de HDL a los seis meses del primer valor tomado.

Se realizó una base de datos en la que se registró las siguientes variables: sexo, edad, fecha de ingreso a UPS, primer control, segundo control, tercer control, peso, talla, índice de masa corporal (IMC), antecedentes de hipertensión arterial (HTA), diabetes tipo 1, diabetes tipo 2, dislipemia, tratamiento con estatinas, status fumador (fumadores activos, fumadores en cesación, ex fumadores y no fumadores) y niveles de HDL.

El status de fumador fue definido como:

a) Fumador

- Activo: aquel que fuma al menos 1 cigarrillo diario u ocasionalmente en los últimos 6 meses.

- Fumador en cesación: fumadores en proceso de abandono del cigarro o habiendo sido fumador, se encuentra en abstinencia menor a 6 meses

b) Exfumador: aquel que habiendo sido fumador se encuentra en abstinencia en últimos seis meses.

c) No fumador: nunca fumo o fumó $<100$ cigarrillos en toda su vida.

Los niveles de colesterol HDL se definió como:

- Bajo: hasta $39.99 \mathrm{mg} / \mathrm{dL}$

- Normal: igual o mayor de $40 \mathrm{mg} / \mathrm{dL}$

Los controles a los pacientes son realizados cada tres meses en la UPS definiéndose: Ingreso a UPS como la primera consulta; primer control, el realizado a los tres meses a partir del ingreso; segundo control, el realizado a los seis meses de su ingreso; tercer control, el realizado a los nueve meses de su ingreso a la UPS.

En la base de datos se analizó: (a) las principales características demográficas de los pacientes, (b) la prevalencia de fumadores activos, en cesación, exfumadores y no fumadores, (c) el nivel lipídico deHDL (d) comparación del status de fumador y niveles de HDL inicial (e) comparación del status de fumador y niveles de HDL a los seis meses (f)comparación del status de fumador y niveles de HDL inicial versus status de fumador y niveles de HDL a los seis meses de seguimiento en la UPS.

Para el análisis longitudinal se seleccionó estrictamente a los pacientes en función de si tenían o no el valor de HDL inicial o al primer control y a los seis meses a partir de estos, que coincidía con el segundo o tercer control. El status de fumador era registrado en cada control, y los datos analizados fueron los que coincidían con los valores de HDL al ingreso o primer control y a los 6 meses a partir de los mismos.

Los resultados se presentan en frecuencias y proporciones para las variables cualitativas y cálculo de la media y desviación estándar (DS) para las variables cuantitativas. El test estadístico utilizado para la comparación de proporciones fue chi-cuadrado (X2) y test de student (t-test) para la comparación medias, considerándose estadísticamente significativo una $p<0.05$. El programa estadístico utilizado fue IBM SPSS statistics 24 (versión gratuita).

\section{Resultados}

De un total de 542 registros clínicos de pacientes atendidos en la UPS en el periodo comprendido entre 01/01/2011 y 31/12/2015, 97 registros médicos cumplieron los criterios de inclusión y exclusión (Figura 1). 


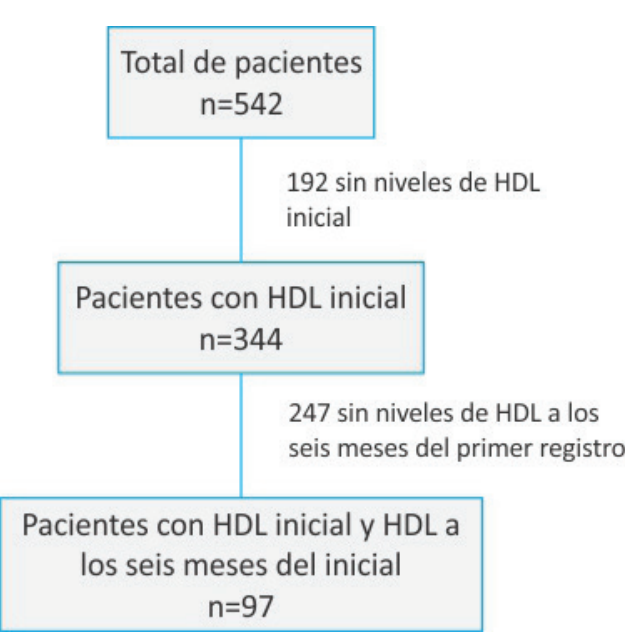

De los 97 pacientes, el $64.9 \%$ eran hombres y el $35.1 \%$ mujeres con una edad media de 58.02 años. El IMC medio fue $29.05 \mathrm{Kg} / \mathrm{m} 2$, destacándose que el $36.5 \%$ de los pacientes tenía sobrepeso y el $39.5 \%$ algún tipo de obesidad. En relación a las principales comorbilidades el $64.9 \%$ tenía HTA, el $53.6 \%$ dislipemia, el $21.6 \%$ diabetes tipo 2, el $1 \%$ diabetes tipo 1. Dentro de los antecedentes familiares el $21.6 \%$ tenían antecedentes de coronariopatía y el $10.3 \%$ de dislipemia familiar. El $100 \%$ de los pacientes tenían indicación de estatinas (Tabla 1).

\begin{tabular}{|c|c|c|c|c|}
\hline \multicolumn{2}{|r|}{ Media } & Desviación estándar & Minimo & Máximo \\
\hline Edad (años) & 58,02 & 7,97 & 36 & 70 \\
\hline Peso (Kg) & 80,02 & 14,81 & 46,20 & 133,25 \\
\hline Talla (cm) & 165,92 & 8,70 & 149 & 190 \\
\hline IMC (kg/m2) & 29,05 & 4,91072 & 20,53 & 47,51 \\
\hline & & Frecuencia & \multicolumn{2}{|c|}{ (\%) } \\
\hline \multirow{2}{*}{ Sexo } & Masculino & $63 / 97$ & \multicolumn{2}{|l|}{64,9} \\
\hline & Femenino & $34 / 97$ & \multicolumn{2}{|l|}{35,1} \\
\hline \multirow{5}{*}{ Distribución por IMC } & Peso normal & 23 & \multicolumn{2}{|l|}{24,0} \\
\hline & Sobrepeso & 35 & \multicolumn{2}{|l|}{36,5} \\
\hline & Obesidad de clase 1 & 32 & \multicolumn{2}{|l|}{33,3} \\
\hline & Obesidad de clase 2 & 3 & \multicolumn{2}{|l|}{3,1} \\
\hline & Obesidad de clase 3 & 3 & \multicolumn{2}{|l|}{3,1} \\
\hline \multicolumn{2}{|l|}{ HTA } & 63 & \multicolumn{2}{|l|}{64,9} \\
\hline \multicolumn{2}{|l|}{ Diabetes tipo 1} & 1 & \multicolumn{2}{|l|}{1,0} \\
\hline \multicolumn{2}{|l|}{ Diabetes tipo 2} & 21 & \multicolumn{2}{|l|}{21,6} \\
\hline \multicolumn{2}{|l|}{ DLP } & 52 & \multicolumn{2}{|l|}{53,6} \\
\hline \multicolumn{2}{|l|}{ AF. DLP } & 10 & \multicolumn{2}{|l|}{10,3} \\
\hline \multicolumn{2}{|l|}{ AF. Coronariopatía } & 21 & \multicolumn{2}{|l|}{21,6} \\
\hline \multicolumn{2}{|c|}{ Tratamiento con estatinas } & 97 & \multicolumn{2}{|l|}{100} \\
\hline
\end{tabular}

En relación al status de fumador al inicio en la UPS, el $48.5 \%$ de los pacientes eran fumadores, de los cuales el $12.4 \%$ eran fumadores activos y el $36.1 \%$ estaban en cesación. El 29.9\% eran ex fumadores y el $21.6 \%$ no fumadores. A los seis meses de seguimiento se evidenció un aumento del porcentaje de exfumadores a 57.7\%, y fumadores a 15.5\% (Grafico 1). 


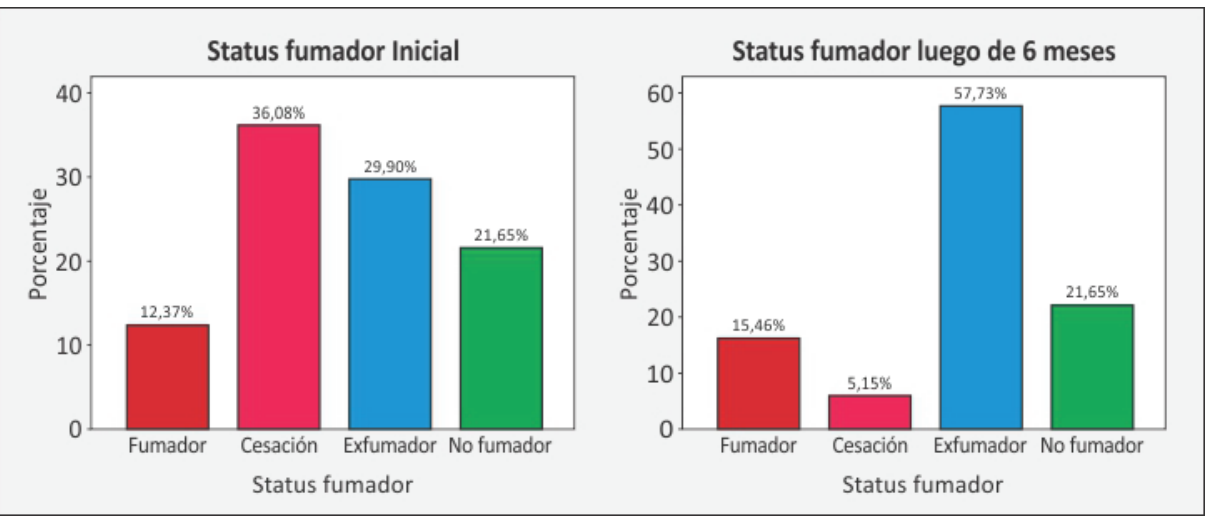

El 53.6\% de pacientes tenía un HDL inicial bajo y el 46.4\% tenía HDL normal. A los 6 meses, se observó una disminución al $41.2 \%$ de pacientes con HDL bajo y un aumento al $58.8 \%$ de los pacientes con colesterol HDL normal (Grafico 2).

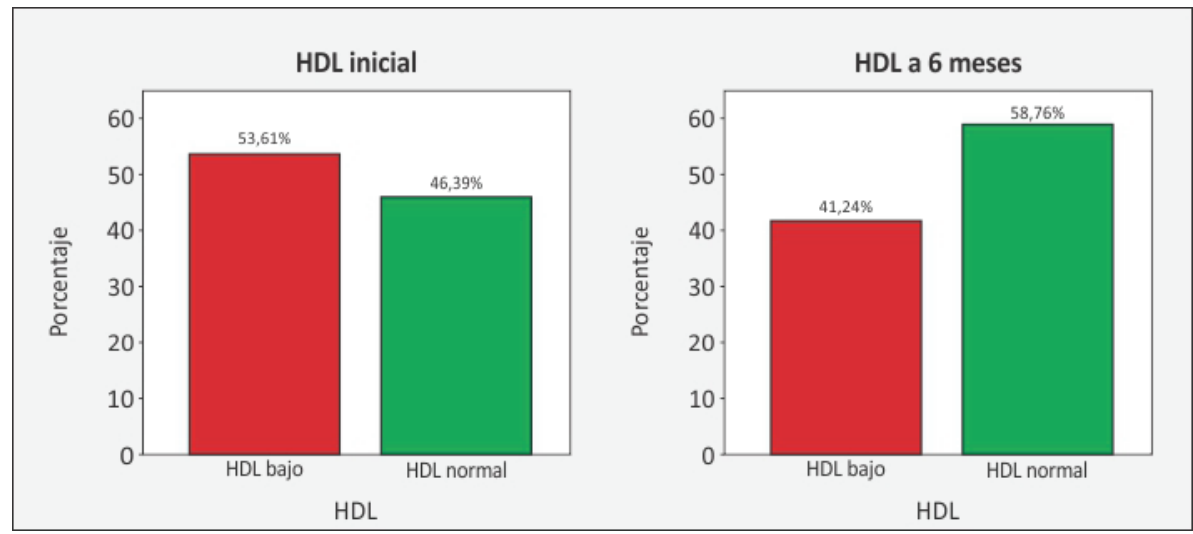

Al realizar la combinación de variables entre el status de fumador y niveles de HDL inicial, se encontró que el $58.3 \%$ de los fumadores activos, el $57.1 \%$ de los fumadores en cesación, el $55.2 \%$ de los exfumadores y el $42.9 \%$ los no fumadores tenían HDL bajo. A los seis meses se observó que el $60 \%$ de los fumadores activos presentaban HDL bajo, mientras que el $40 \%$ de los fumadores en cesación, el $42,1 \%$ de los ex fumadores y $29,6 \%$ de los no fumadores presentaba HDL bajo (Gráfico 3).

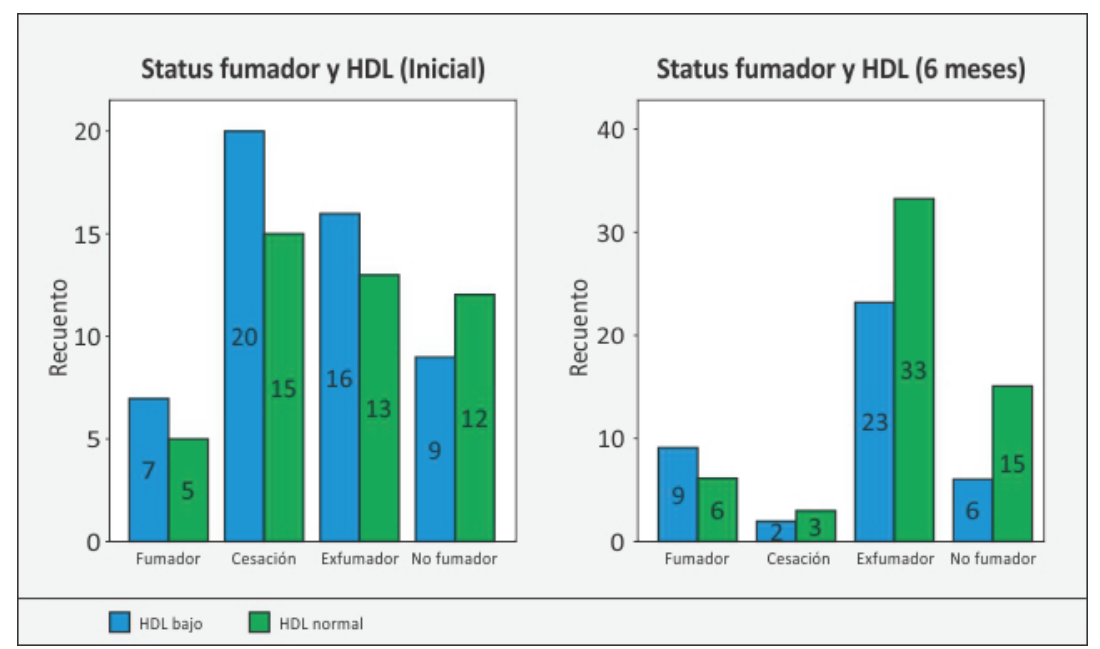

Estos resultados no fueron estadísticamente significativos, con un valor de $p$ 0,732 al inicio y $\mathrm{p} 0,311$ a los 6 meses.

Dado que al inicio en la UPS, la mayoría de los fumadores estaba en cesación y que a los 6 meses estos pasaron a ser ex fumadores, se comparó la media de HDL de los fumadores en cesación iniciales versus los exfumadores a los 6 meses, observándose un aumento de 
los valores de $40.17 \mathrm{mg} / \mathrm{dl}$ a $43.84 \mathrm{mg} / \mathrm{dl}$ respectivamente, con una $\mathrm{p}$ de 0.054 que roza la significancia estadística (Gráfico 4).

Gráfico 4: Media según status de fumador y niveles de HDL inicial y a los 6 meses

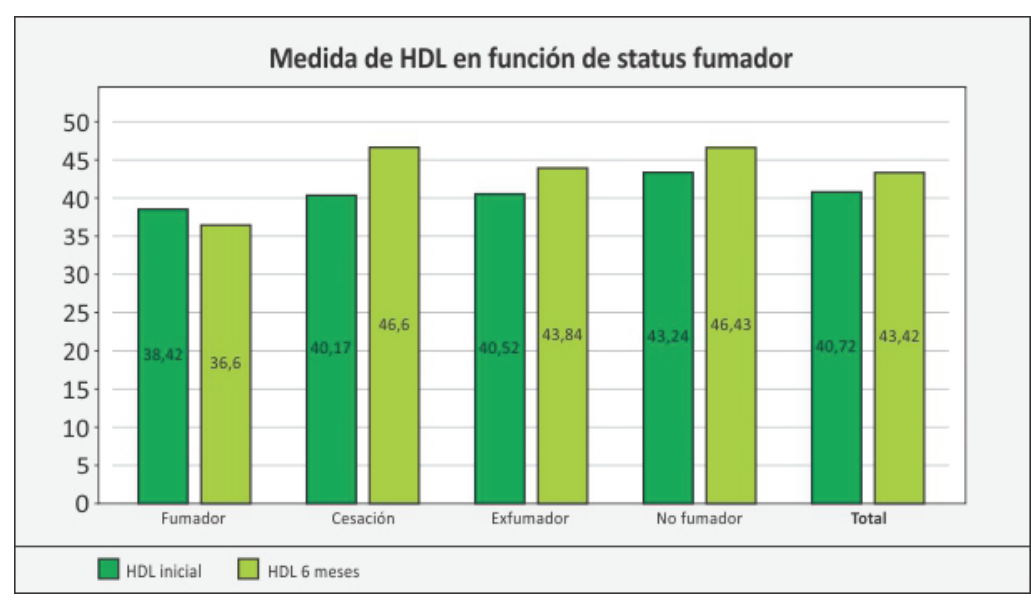

La media total de los valores de HDL inicial fue de $40.72 \mathrm{mg} / \mathrm{dl}$ y a los 6 meses de $43.42 \mathrm{mg} /$ $\mathrm{dl}$, que al compararlos dio un valor de p 0.003 estadísticamente significativo (Tabla 2).

\begin{tabular}{l|l|l|l|l|l|l|l} 
& \multicolumn{3}{|c|}{ HDL inicial } & \multicolumn{3}{c|}{ HDL 6 meses } & Valor p \\
\hline Status & Media & N & DS & Media & N & DS & \\
\hline Fumador & 38.42 & 12 & 13.180 & 36.60 & 15 & 9.560 & \\
\hline Cesación & 40.17 & 35 & 11.155 & 46.6 & 5 & 16.334 & \\
\hline Ex fumador & 40.52 & 29 & 13.437 & 43.84 & 56 & 11.468 & \\
\hline No fumador & 43.24 & 21 & 9.617 & 46.43 & 21 & 10.847 & \\
\hline Total & $\mathbf{4 0 . 7 2}$ & $\mathbf{9 7}$ & $\mathbf{1 1 . 7 5 1}$ & $\mathbf{4 3 . 4 2}$ & $\mathbf{9 7}$ & $\mathbf{1 1 . 5 8 4}$ & $\mathbf{0 , 0 0 3}$
\end{tabular}

\section{Discusión}

Nuestro trabajo permitió conocer datos importantes de pacientes con cardiopatía isquémica con revascularización miocárdica atendidos en una UPS.

En nuestro estudio prevaleció el sexo masculino en relación al femenino. Esta tendencia es similar a la prevalencia de fumadores según el sexo observado a nivel nacional ${ }^{(6)}$. La edad de presentación de cardiopatía isquémica fue antes de los 60 años en la mayoría de los casos, probablemente relacionado a varios factores de riesgo predisponentes, como tabaquismo, HTA, diabetes, dislipemia, sobrepeso y obesidad observada en nuestro grupo de pacientes.

Al analizar el status de fumador, se encontró que los fumadores presentaban niveles bajos de HDL en comparación a los exfumadores y no fumadores. Destacamos que los pacientes que continuaron fumando mantuvieron niveles de HDL bajo, pese a las medidas higiénico dietéticas y medicamentosas recibidas en la UPS. Estos resultados se correlacionan con otros estudios ${ }^{(9-}$ ${ }_{11,15-17)}$ en los cuales se demuestra el efecto negativo del tabaquismo sobre el HDL y la asociación al aumento de riesgo cardiovascular.

Aquellos pacientes que al ingreso a la UPS estaban en cesación, la mayoría dejó de fumar, observándose un aumento de los exfumadores a los 6 meses. No todos los fumadores en cesación lograron dejar de fumar, como se ve reflejado en nuestro estudio dado el aumento de fumadores activos a los 6 meses, por lo que se debería reforzar las medidas de cesación del tabaquismo. A excepción de los fumadores activos, los fumadores en cesación y ex fumadores aumentaron y normalizaron los niveles de HDL a los seis meses de seguimiento, la diferencia de este aumento rozaba la significancia estadística. Nuestros resultados se parecen a los de otros estudios que demuestran que el dejar de fumar eleva los niveles de HDL ${ }^{(9,10,18-20)}$.

Al comparar las medias totales de HDL inicial y HDL a los 6 meses, se encontró valores más altos de HDL a los seis meses, siendo estos resultados estadísticamente significativos. Esta diferencia en los valores se podría explicar por un lado a la cesación del tabaquismo en la mayoría de los pacientes, y por otro lado debido a las medidas higiénicas dietéticas y medicamentosas realizadas en la UPS. 
Como fortalezas de este este estudio destacamos los controles trimestrales a los pacientes en la UPS, pudiendo de esta manera realizar el seguimiento del status de fumador y la realización de la paraclínica para constatar los niveles de HDL. Entre las debilidades destacamos la cantidad de pacientes, ya que corresponde casi la quinta parte del número total de pacientes atendidos en la UPS debido a que no contaban con registros de HDL iniciales y a los seis meses que fue el criterio de inclusión. Consideramos que el número pequeño de pacientes influyó para que los resultados tengan una tendencia favorable pero no sean estadísticamente significativos, por lo que hace pensar que se debe analizar una muestra más grande, para poder extrapolar los resultados a toda la UPS. Otra de las debilidades fue que no se pudo realizar el análisis de otras variables, su vinculación e incidencia clara en nuestros resultados, como el tipo de dieta, el tiempo de ejercicio y las dosis de estatinas debido a que estos datos estaban poco detallados para el análisis, aspectos que se tomaran en cuenta para futuros estudios.

\section{Conclusiones}

En pacientes portadores de cardiopatía isquémica con revascularización miocárdica atendidos en la UPS, la frecuencia de fumadores fue de $48,5 \%$, de exfumadores $29,9 \%$ y no fumadores $21,6 \%$. Los niveles de HDL fueron bajos en los fumadores, confirmándose la relación entre el tabaquismo y la disminución del HDL. Encontramos una tendencia a aumentar los valores de HDL en los fumadores que se convirtieron en exfumadores, por lo que podemos afirmar que la cesación del tabaquismo es una conducta importante para la elevación de los niveles de HDL. La comparación de HDL total inicial y HDL total a los 6 meses demostró ser estadísticamente significativo. Entre los factores no analizados pero que pudieron haber influido positivamente en la elevación del HDL son las medidas higiénico dietéticas y tratamiento farmacológico recibido en la UPS.

\section{Agradecimientos}

Agradecemos a la Clínica Médica $\nabla A \bigotimes$, su directora Prof. Dra. Gabriela Ormaechea y a los médicos integrantes de la Unidad de Tabaquismo y de la Unidad de Prevención Secundaria de la Clínica Médica $\triangle A \otimes$ del Hospital de Clínicas por la colaboración, el registro y la facilitación de los datos contenidos en las historias médicas electrónicas.

\section{Financiación}

El estudio no contó con financiación específica.

\section{Conflictos de interés}

Los autores declaramos no poseer conflictos de interés alguno.

\section{Bibliografía}

1- Organizacion Mundial de la Salud. Enfermedades Cardiovasculares [Internet]. OMS; 2015. [cited 2016 May 17]. Available: http://www.who.int/mediacentre/factsheets/fs317/es/

2- Gambogi R, Baldizzoni M, Saona G, Niggemeyer A, Wald M, Nigro S, et al. Efectividad de un programa de prevención secundaria cardiovascular en Uruguay. Rev Méd Urug. 2013;29(2):74-84.

3- Uruguay. Comisión Honoraria para la Salud Cardiovascular. Morbilidad y Mortalidad por enfermedades cardiovasculares en el Uruguay 2013 - 2014. Montevideo: Área de Epidemiología y Estadística; 2015.

4- Sandoya E, Bianco E. Mortalidad por tabaquismo y por humo de segunda mano en Uruguay. Rev Urug Cardiol. 2011; 26(1):201-6.

5- Machado F, Bianco E, Nuñez E, Torres F, Sandoya E. Ingresos por infarto agudo de miocardio en los cuatro años siguientes a la implementación de la prohibición de fumar en espacios cerrados. Rev Urug Cardiol. 2015;30(3):280-5.

6- Instituto Nacional de Estadistica. Principales resultados encuesta continua de hogares (ECH) 2014. Montevideo; 2014.

7- Control del Tabaquismo - Ley No 18.256 [Internet]. Uruguay: Camara de Senadores y Representantes. Poder Legislativo; 2016. [cited 2016 Jul 24]. Disponible en: https://legislativo.parlamento.gub.uy/ temporales/leytemp9439905.htm

8- Vázquez H, Burdiat G, Alonso P, Sandoya E, Tejada J. Control del riesgo cardiovascular postinternación en pacientes coronarios. Rev Uruguaya Cardiol. 2011;26(2):108-14. 
9- Gepner AD, Piper ME, Johnson HM, Fiore MC, Baker TB, Stein JH. Effects of smoking and smoking cessation on lipids and lipoproteins: Outcomes from a randomized clinical trial. Am Heart J. 2011;161(1):145-51.

10- Johnson HM, Gossett LK, Piper ME, Aeschlimann SE, Korcarz CE, Baker TB, et al. Effects of smoking and smoking cessation on endothelial function: 1-year outcomes from a randomized clinical trial. J Am Coll Cardiol. 2010;55(18):1988-95.

11- Giunzioni I, Bonomo A, Bishop E, Castiglioni S, Corsini A, Bellosta S. Cigarette smoke condensate affects monocyte interaction with endothelium. Atherosclerosis. 2014;234(2):383-90.

12- Sandoya E, Sebrié E, Bianco E, Araújo O, Correa A, Davyt O, et al. Impacto de la prohibición de fumar en espacios cerrados sobre los ingresos por infarto agudo de miocardio en Uruguay. Rev.méd Urug. 2010;26(4):206-15.

13- Perk J, De Backer G, Gohlke H, Graham I, Reiner Ž, Verschuren M, et al. European Guidelines on cardiovascular disease prevention in clinical practice (version 2012). The Fifth Joint Task Force of the European Society of Cardiology and Other Societies on Cardiovascular Disease Prevention in Clinical Practice (constituted by representatives of nine societies and by invited experts). Eur Heart J. 2012;33(13):1635-701.

14- Sandoya E. Impacto del tabaquismo y el humo de segunda mano en la salud cardiovascular. Arch Med interna. 2011;26 (1):29-38.

15- Campbell SC, Moffatt RJ, Stamford BA. Smoking and smoking cessation-The relationship between cardiovascular disease and lipoprotein metabolism: A review. Atherosclerosis. 2008;201(2):225-35.

16- Gossett LK, Johnson HM, Piper ME, Fiore MC, Baker TB, Stein JH. Smoking intensity and lipoprotein abnormalities in active smokers. J Clin Lipidol. 2009;3(6):372-8.

17- Freeman DJ, Griffin BA, Murray E, Lindsay GM, Gaffney D, Packard CJ, et al. Smoking and plasma lipoproteins in man: effects on low density lipoprotein cholesterol levels and high density lipoprotein subfraction distribution. Eur J Clin Invest. 1993;23(10):630-40.

18- Forey BA, Fry JS, Lee PN, Thornton AJ, Coombs KJ. The effect of quitting smoking on HDL-cholesterol - a review based on within-subject changes. Biomark Res. 2013;1(1):26.

19- Maeda K, Noguchi Y, Fukui T. The effects of cessation from cigarette smoking on the lipid and lipoprotein profiles: A meta-analysis. Prev Med (Baltim). 2003;37(4):283-90.

20- Noh J-M, Lee S-H, Kim H-W, Yang H-S. Changes in the Serum Level of High Density Lipoproteincholesterol after Smoking Cessation among Adult Men. Korean J Fam Med. 2012;33(5):305-10.

21- Fondo Nacional de Recursos. Programa de prevención secundaria cardiovascular [en linea]. 2017 [acceso 27/09/2017]. Disponible en: http://www.fnr.gub.uy/descripcion_cardio

\section{Aporte cada autor al trabajo}

Darwin Cordovilla: Participación 50\%

Laura Llambi: Participación 25\%

Selva Romero: Participación 25\% 